\title{
Therapeutic Approaches to Dysphagia Treatment in Parkinson Disease: A Review
}

\author{
Ali Akbar Dashtelei ${ }^{1}$, Ahmad Reza Khatoonabadi (iD) ${ }^{1, *}$ and Jalal Bakhtiari ${ }^{2}$ \\ ${ }^{1}$ Speech Therapy Department, Tehran University of Medical Sciences, Terhan, Iran \\ ${ }^{2}$ Speech Therapy Department, Neuromuscular Rehabilitation Research Center, Semnan University of Medical Sciences, Semnan, Iran \\ "Corresponding author: Speech Therapy, Tehran University of Medical Sciences, Terhan, Iran. Email: a.r.khatoonabadi@gmail.com \\ Received 2017 December 09; Revised 2019 May 12; Accepted 2019 June 20.
}

\section{Abstract}

Context: Dysphagia is one of the common symptoms observed in more than $80 \%$ of patients with Parkinson disease (PD). Dysphagia is a potentially harmful feature that leads to the reduction of the quality of life, malnutrition, and aspiration pneumonia, which is one of the main causes of death in patients with PD. The current study aimed at reviewing therapeutic approaches to dysphagia treatment in PD.

Evidence Acquisition: MEDLINE literature through PubMed until 2017 was reviewed.

Results: Studies on the dysphagia treatment approaches in PD are very diffuse. These studies include the conventional rehabilitation of dysphagia, as well as behavioral-instrumental and electrophysiological treatments. Despite the usefulness of these treatments, due to the poor quality of such studies, the generalization of their results is limited.

Conclusions: It appears that a combination of treatments provides satisfactory results. Therefore, it is necessary to use new treatment methods in addition to the conventional treatments.

Keywords: Parkinson Disease, Dysphagia, Rehabilitation, Treatment

\section{Context}

Parkinson disease (PD) is one of the progressive disorders of the nervous system caused by the progressive reduction of dopamine producing cells in ventrolateral part of parse compacta located in substantia nigra of midbrain $(1,2)$. The prevalence of PD in people over 60 years old is about $1 \%$ (3). PD has a set of motor and non-motor symptoms. The main motor symptoms include bradykinesia, tremor, rigidity, and posture instability (4). Non-motor manifestations in such patients also include sleep disorder, depression, anxiety, psychosis, sexual problems, cognitive impairments including learning disorder and disorders of automatic nervous system such as respiratory problems. One of the most common and important symptoms in patients with PD is difficulty in swallowing or dysphagia. Dysphagia is taken into consideration since it reduces the quality of life, and even can lead to the death in patients with PD due to aspiration pneumonia $(4,5)$. Therefore, the current study aimed at reviewing the therapeutic approaches to dysphagia treatment in PD.

\section{Evidence Acquisition}

MEDLINE literature through PubMed until 2017 was reviewed using the following keywords: swallowing and PD, swallowing disorder and PD, dysphagia and PD, therapeutic approaches to dysphagia and PD, aspiration pneumonia and dysphagia in PD. Furthermore, articles on the efficacy of new electrophysiological treatment for dysphagia improvement were investigated.

\section{Results}

\subsection{Normal Swallowing}

Normal swallowing includes four phases: oral preparatory, oral transfer, pharyngeal, and esophageal (6) that are physiologically continuous. Swallowing biomechanics shows how the integration of a phase depends on integration in other phases. Oral preparatory and oral transfer phases considered as voluntary phases are performed by the activity of the lips, teeth, jaws, and tongue. In oral preparatory phase, the lips prevent the material from getting out of the mouth and the jaw and teeth help form the 
bolus with coordinated movements (7). In the oral transfer phase, the bolus is transferred to the oropharynx area by the wave movements of the tongue. The pharyngeal phase is an involuntary phase, which begins with the activation of swallowing reflex (8). Six events occur in the pharyngeal phase of swallowing: (1) velopharyngeal valve closure, (2) hyolaryngeal complex elevation, (3) laryngeal glottic closure, (4) opening upper esophageal sphincter in order to transfer the bolus to the esophagus, (5) retraction of the base of tongue, and (6) contraction of the posterior part of pharyngeal wall (6). The final phase of swallowing is esophageal phase that the food is transferred to the stomach by esophageal peristaltic movements after passing through the upper esophageal sphincter (UES) (9). To perform these sequential and complex swallowing movements, different nervous centers from different areas including the primary and secondary sensory-motor cortical areas of both hemispheres, anterior insular cortex, frontal lobe operculum, the anterior cingulate, the posterior parietal cortex, supplementary motor area, medulla oblongata, and the inferior part of pons are involved (1014).

\subsection{Oropharyngeal Dysphagia in Parkinson Disease}

Dysphagia is observed in any of the swallowing phases. Dysphagia is considered as a problematic characteristic in patients with Parkinson disease (PD) and its prevalence is different in various studies due to different stages of the disease, the unknown nature of swallowing in PD, and the type of swallowing assessment methods. This prevalence is reported $30 \%$ to $100 \%(15,16)$.

Oropharyngeal dysphagia has various medical, psychosocial, and even economic complications in such patients. The most important medical complications of dysphagia include aspiration pneumonia, dehydration, and malnutrition that also lead to death of patients in some cases, if diagnosis and treatment are not timely. Psychosocial complications that could have a negative impact on the quality of life include depression and social isolation (17). Swallowing problem in patients with PD is observed in almost all swallowing phases, but the role of speechlanguage pathologist is more in the assessment and treatment of oral (oral preparatory and transfer) and pharyngeal phases. Symptoms of the disorder in the oral phase include inadequate bolus preparation, anterior leakage, and drooling. Also, other symptoms consist of repetitive A-P movement of bolus in the oral cavity especially tardive in tongue movements, motor delay, and tongue tremor. Premature spillage of food to pharyngeal area during the oral preparatory phase, multiple swallowing, and oral residue are other problems observed in the oral phase $(18,19)$.

Dysphagia in pharyngeal phase is observed as delay in swallowing reflex, reduced elevation of hyolaryngeal complex, reduced pharyngeal sensation, and thus reduced voluntary cough, food residual in vallecula and pyriform sinuses, and reduced speed in spontaneous swallowing (16, 19, 20). Also, glottic closure is performed with delay, which leads to aspiration and subsequent aspiration pneumonia. Swallowing problems in the esophageal phase is also observed as spasm, which reduces the food transfer and multiple contractions (19).

\subsection{Pathophysiology of Dysphagia in Patients with Parkinson Disease}

Swallowing motor control is bilaterally performed in the cerebral cortex. Evidence show that there is a type of functional asymmetry in motor network of swallowing between the hemispheres to control the swallowing functions (21). In patients with PD and dysphagia, the pathophysiology of dysphagia is still unknown. On one hand, the lack of fixed answer in improving the function of swallowing of patients with PD to dopaminergic drugs, and on the other hand, the lack of a specific relationship between dysphagia severity and duration of PD or general motor impairment shows that disturbances in the nondopaminergic network can play a key role in PD dysphagia $(17,22)$.

In addition, evidence shows that in PD, damage to control basal ganglia nuclei leads to disturbance in voluntary movements of swallowing (oral phase), damage to the central pattern generator swallowing parts in the medulla oblongata, the dorsal motor nucleus of the vagus nerve as well as the peripheral nervous system leading to dysphagia. Also, pedunculopontine tegmental nucleus, which is reinforcing the nucleus of the inputs of taste path nucleus, is one of the other essential components of the swallowing function in the medulla oblongata, which play role in inhibiting pallidum inputs and are damaged in the progressive and a neurodegenerative diseases $(10,23)$. Recently, neuroimaging studies show that cortical area such as the primary sensory-motor cortex, integrated sensory-motor area, insula, the anterior cingulate, and supplementary motor area (SMA) in addition to the brain stem play an important role in the act of swallowing $(6,24)$. MEG evidence in the assessment of swallowing-related cortical activities in patients with PD show that local activity in the parts of caudolateral from the premotor and primary sensorymotor cortex as well as the inferiolateral part of parietal 
lobe was observed in patients without dysphagic complications, but SMA activity is clearly reduced in such patients compared to that of the healthy people; while there is a significant reduction in activity levels associated with the task in patients with dysphagic Parkinson. Particularly, most of the damage is related to the primary sensory-motor area in patients with dysphagic Parkinson. In fact, it can be observed that in the early stages of progressive PD with the advancement of neuronal degeneration, first, secondary sensory-motor cortex is damaged, while damage to the primary sensory-motor area is observed in the final stages of the disease (17).

\subsection{Methods of Assessment and Specific Tests for Swallowing in Patients with Parkinson Disease}

Assessment and diagnosis of dysphagia in patients with PD similar to the other pathologies include clinical and instrumental assessments that either self-reported questionnaires (subjective assessment) or instrumental assessment (objective assessment) can be used. Dysphagia in the subjective assessment is much less identifiable than the objective assessment; therefore, less than 50\% of patients with PD and identified dysphagia by an instrumental assessment are aware of their swallowing problem (16). Two instruments as the Munich dysphagia test (MDTPD) and swallowing disturbance questionnaire (SDQ) are specifically used to assess and identify dysphagia in patients with PD. MDT-PD focuses on the early detection of dysphagia symptoms in the early stages of PD, while SDQ focuses on detecting aspiration and the existing dysphagia; $20 \%$ to $40 \%$ of patients with dysphagic Parkinson are aware of their dysphagia problem and less than $10 \%$ report their swallowing problems. Therefore, the importance of these questionnaires is more obvious in early diagnosis of dysphagia (19).

The fiberoptic endoscopic evaluation of swallowing (FEES) and video fluoroscopy swallowing study (VFSS) can be mentioned as standard diagnostic tools of dysphagia that their results are combined with the penetrationaspiration scale (PAS). FEES and VFSS are used to assess the oral and pharyngeal phases of swallowing and more than $80 \%$ of patients with PD show the symptoms of dysphagia during their disease according to the findings these tools. Also, high resolution manometry (HRM) method is useful to investigate the swallowing problems in esophageal phase as well as the clinical identification of non-obvious swallowing problems in early stages of the disease (19).

As mentioned above, dysphagia could have a negative effect on the quality of life of patients with PD by creat- ing the social and functional constraints and mood disorders. Therefore, one of the important factors associated with swallowing is quality of life assessed by the speech and language pathologists as a criterion for treatment progression. There are limited questionnaires to assess the quality of life associated with swallowing, which include SWAL-QOL, MDADI, DHI, and EAT-10 questionnaires (1, 2). According to the literature, SWAL-QOL and MDADI questionnaires are used to investigate the effect of dysphagia on the quality of life in patients with PD (11). The SWAL-QOL questionnaire was developed in 2000 with 11 subscales and 44 items. The MDADI questionnaire was also designed in 2001 and has 20 items.

\subsection{Dysphagia Treatment Methods in Patients with Parkinson Disease}

Although there is no specific underlying pathophysiology about the cause of dysphagia in patients with PD, favorable results can be obtained based on the existing findings if the treatment of dysphagia is according to the underlying pathophysiology (12). Very limited studies are conducted on the effectiveness of interventional dysphagia in patients with PD. Treatments of dysphagia in patients with PD can be classified as conventional and new treatments. Treatments of dysphagia include surgical, pharmaceutical, and rehabilitation approaches; while new treatments emphasize behavioral-instrumental approaches such as expiratory muscle strengthen training (EMST), video-assisted swallowing therapy (VAST), and electrophysiological treatments such as surface electrical stimulation(SES) and deep brain stimulation (DBS). These methods are categorized in Table 1.

\begin{tabular}{lc}
\hline Table 1. Dysphagia Treatment Methods in Patients with Parkinson Disease \\
\hline Treatment & Treatment Method \\
\hline Conventional treatments & Surgical, Pharmaceutical, Rehabilitation \\
New treatments & EMST, VAST, SES, DBS \\
\hline
\end{tabular}

Abbreviations: EMST, expiratory muscle strengthen training; DBS, deep brain stimulation; SES, surface electrical stimulation; VAST, video-assisted swallowing therapy.

\subsection{Surgical Treatment}

Limited studies are reported about surgical treatment. In these studies, the problem in most of the patients was in esophageal phase of swallowing that was related to bad function of cricopharyngeal valve. Therefore, surgical treatment was performed in such patients by removing 
the cricopharyngeal valve. Manometry and VFSS test before surgery were used in these studies, but no instrumental assessment was performed after surgery and only the patients' comments showed the efficacy of surgical treatment in complete improvement of the dysphagia problem in patients with $\mathrm{PD}(13)$.

\subsection{Pharmaceutical Treatment}

Pharmaceutical treatment is usually performed by the prescription of L-dopa and injection of botulinum toxin in the parotid glands (14). The effects of pharmaceutical treatment on improving motor functions of patients with PD are well demonstrated. However, contradictory results are reported on the effectiveness of pharmaceutical treatment on the swallowing function of patients with PD. Some studies indicate the effect of pharmaceutical treatment on reducing the symptoms of dysphagia, while others indicate no effect $(10-12,14,19-26)$. Also, some (or several) studies show that botulinum toxin injection (BT-A) only leads to sialorrhoea decrease in patients with PD, but it has no effect on improving the swallowing function according to VFSS assessments (14).

\subsection{Rehabilitation Treatment of Swallowing}

Newer therapeutic methods and approaches are used in various pathologies since 1980 s, considering the assessment and treatment of oropharyngeal dysphagia as a specialized field of clinical activity of speech and language pathologists; and the effectiveness of many of these methods in various diseases are investigated and confirmed. Rehabilitation treatments in PD are selected according to signs and symptoms of dysphagia (12). Rehabilitation treatments of swallowing are considered as alternative to pharmaceutical treatment and include swallowing exercises, compensatory maneuvers, positional changes, LeeSilverman voice treatment (LSVT), food modify, chewing, etc. $(14,19,27)$. Several studies show the effectiveness of compensatory and rehabilitation methods on improving dysphagia in patients with PD $(13,28-31)$. But, the results of these studies cannot be compared or generalized due to the methodological defects such as heterogeneous population of the studied patients, lack of control group, lack of randomly assignment of patients, different treatment methods, and different output criteria (19). Conventional treatments have a negative impact on quality of life. For example, prescribing compensatory strategies (such as tucking the chin or thickened liquids) are likely to adversely impact the patient's quality of life (32). No randomized clinical trials were obtained based on these treatments.

\subsection{Behavioral-Instrumental Treatments}

Unfortunately, very limited valid interventional studies are conducted on dysphagia of patients with PD. Given that effective function of the oropharyngeal neuromuscular system and motor and coordination skills plays a major role in safe swallowing, these variables are therefore considered as the targets of rehabilitation exercises. Thus, behavioral-instrumental techniques are used to improve these functions $(14,19)$. In general, randomized clinical trials on patients with PD included investigations on the effectiveness of EMST and VAST on swallowing function and the effectiveness of these treatments was assessed in the current study $(33,34)$.

\subsection{EMST}

EMST leads to increase the power of sub-mental muscles and consequently increase the movements of hayolarynx complex. The effectiveness of EMST on dysphagia in patients with PD compared to the sham group was investigated in a study. In this study, patient received treatment 25 times a day, five days a week for four weeks, and then the patient was assessed by a VFSS post-test and PAS scale score was recorded. Results indicated improvement of dysphagia and reduction of aspiration after EMST treatment in the studied patient (33). Despite the effectiveness of this treatment, the results cannot be generalized to the population of patients with dysphagia due to the limited range of disease and severity of dysphagia in subjects (3).

\subsection{VAST}

VAST treatment is relied on a mechanism to provide visual clues that includes observation of the normal swallowing process and the damaged swallowing of the patient himself using the FEES (4). The effectiveness of the VAST method was investigated compared to the conventional treatment. In the study, VAST treatment was performed as six sessions of observation of the normal swallowing process as well as observation of the swallowing process of the patient himself through FEES. The conventional treatment was performed in the control group, and VAST treatment, in addition to the conventional treatment, was performed in the experimental group, and swallowing function before and after the treatment was assessed by FEES test; the quality of life associated with dysphagia was also assessed by SDQ test. According to the findings of the study, pharyngeal residues reduced in the VAST treatment group and the swallowing-related quality of life scores were improved (34). But since this effectiveness was reported shortly after delivering VAST treatment, the long-term effectiveness of the method is unclear (3). 


\subsection{Electrophysiological Treatment}

Neurogenic dysphagia in patients with neurological diseases may occur with different etiologies (35). Electrical stimulation as a possible treatment for neurological dysphagia was proposed. Due to the better understanding of the neurophysiology ingestion, the number of such studies was higher. Electrical stimulation may be either peripheral or central $(6,12)$. Electrical stimulation of peripheral nerve is the stimulation of the oropharyngeal muscle activity by electrical pulses that are applied through the electrode to the skin (called transcutaneous neuromuscular electrical stimulation or NMES). Central stimulation techniques stimulate peripheral nerves and muscles as well as the cerebral cortex and affect the underline nervous mechanisms of motor function (6). Electrical stimulation in PD is discussed in the following sections.

\subsection{SES}

SES treatment was also performed as randomized clinical trials in patients with PD in two studies $(36,37)$. In these studies, patients were divided into three randomized groups and the treatment was performed daily for 15 days. All three groups received conventional treatment of swallowing. SES treatment was performed in two groups, so that sensory surface stimulation was performed in one group and motor surface stimulation in another group. SES treatment was delivered as the stimulation of submental area. In these studies, VFSS and FEES assessments were used before and after the intervention and the quality of life associated with the patients' swallowing was assessed by SWAL-QOL and MDAD questionnaires. The results of these studies indicated dysphagia improvement as a result of both conventional and SES treatments and there was no significant difference between the two treatment methods $(36,37)$.

\subsection{4. $D B S$}

DBS of the brain is considered as one of the conventional treatment modalities in advanced stages of PD (27). DBS is actually a surgical treatment in which foursquare electrodes are placed bilaterally in one of the places of thalamus, sub-thalamic nuclei, or the inner part of globus pallidus (12). Results indicated no significant clinical improvement in swallowing function in patients with $\mathrm{PF}$, despite improvement of motor functions (27). Emergence of dysphagia can even be the side effect of DBS in patients with $\operatorname{PD}(13,14)$.

\subsection{Alternative Electrophysiological Treatments}

Recently, transcranial direct current stimulation (tDCS) and repetitive transcranial magnetic stimulation (rTMS), because of their noninvasive characters are widely used in the fields of speech and language pathology including dysphagia treatment in patients with stroke. Although the evidence for the effectiveness of these techniques is not enough now, the results of a few studies showed that these techniques are benefited in clinical practice.

\subsection{6. $t D C S$}

The nature of the effectiveness of this treatment in improving dysphagia depends on the type of stimulation. Anodal stimulation facilitates the stimulation of motor cortex; while cathodal stimulation reduces the stimulation of motor cortex (19). Anodal stimulation is usually given to the pharyngeal motor cortex area for 20 - 30 minutes with intensity of $1-2 \mathrm{~mA}$. In dysphagia treatment, tDCS acts in such a way that anodal stimulation increases the stimulation in the pharyngeal motor cortex area and the power of synapses related to the task is increased in swallowing motor network with increasing cortical stimulation in this area and as a result improves the planning function, motor initiation, and swallowing function (38-40).

\subsection{7. rTMS}

Another non-invasive electrical stimulation technique is rTMS. It is used to change and modify cortical, intracortical, subcortical, and spinal structures. In rTMS stimulation, a high current pulse is generated by the coil of wire, which can lead to the depolarization of target neurons. Low-frequency pulses (about $1 \mathrm{~Hz}$ ) have inhibitory effect through slowing the excitability of neurons, while highfrequency pulses ( $\geq 3 \mathrm{~Hz}$ ) have an excitability effect. The rTMS may be used for pharyngeal or esophageal motor cortex in affected or unaffected hemisphere in patients with stroke. Usually, rTMS is used 10 - 20 minutes a day for 5 - 15 days with different intensities between 1 and $5 \mathrm{~Hz}$ or higher, depending on the target (32). However, there was no published evidence for using rTMS in patiens with PD.

\section{Conclusions}

Dysphagia in patients with PD is a common problem; however, the pathophysiology of dysphagia is not definitively determined yet. Therefore, the present therapeutic approaches are unable to certainly treat this problem 
and further studies need to be conducted in this field. According to the current review findings, conventional treatments including compensatory and rehabilitative techniques have the defects such as short-time effects, highcost, and negative impact on quality of life. Thus, using new strategies such as behavioral-instrumental and electrophysiological treatments in combination with current therapies can be more effective. Although the effect of VAST and EMST methods are proven, their long-term effects and potential on patients with different degrees of PD are unknown. Furthermore, regarding new technologies, no clinical trial was conducted on the effectiveness of rTMS and tDCS in swallowing rehabilitation in patients with PD.

It seems that combination approaches is more effective than conventional approaches.

\section{Footnotes}

Authors' Contribution: Ali Akbar Dashtelei: drafting and revision of the manuscript. Ahmad Reza Khatoonabadi: designing of review and revision of the manuscript. Jalal Bakhtiyari: review and revision of the manuscript

Conflict of Interests: The authors declared no conflict of interest.

Ethical Approval: The authors tried to consider all of recent articles in this field without any bias to specific approach.

Funding/Support: There was no funding support for the review study.

\section{References}

1. Klein C, Westenberger A. Genetics of Parkinson's disease. Cold Spring Harb Perspect Med. 2012;2(1). a008888. doi: 10.1101/cshperspect.a008888. [PubMed: 22315721]. [PubMed Central: PMC3253033].

2. Diedrich M, Kitada T, Nebrich G, Koppelstaetter A, Shen J, Zabel C, et al. Brain region specific mitophagy capacity could contribute to selective neuronal vulnerability in Parkinson's disease. Proteome Sci. 2011;9:59. doi: 10.1186/1477-5956-9-59. [PubMed: 21943346]. [PubMed Central: PMC3196908].

3. Tysnes OB, Storstein A. Epidemiology of Parkinson's disease. J Neural Transm (Vienna). 2017;124(8):901-5. doi: 10.1007/s00702-017-1686-y. [PubMed: 28150045].

4. Rodriguez-Oroz MC, Jahanshahi M, Krack P, Litvan I, Macias R, Bezard $E$, et al. Initial clinical manifestations of Parkinson's disease: Features and pathophysiological mechanisms. Lancet Neurol. 2009;8(12):112839. doi: 10.1016/S1474-4422(09)70293-5. [PubMed: 19909911].

5. Hughes AJ, Daniel SE, Kilford L, Lees AJ. Accuracy of clinical diagnosis of idiopathic Parkinson's disease: A clinico-pathological study of 100 cases. J Neurol Neurosurg Psychiatry. 1992;55(3):1814. doi: 10.1136/jnnp.55.3.181. [PubMed: 1564476]. [PubMed Central: PMC1014720].

6. Daniels SK, Huckabee ML. Dysphagia following stroke. Plural Publishing; 2014.
7. Hamdy S, Rothwell JC, Brooks DJ, Bailey D, Aziz Q, Thompson DG. Identification of the cerebral loci processing human swallowing with H2(15)O PET activation. J Neurophysiol. 1999;81(4):1917-26. doi: 10.1152/jn.1999.81.4.1917. [PubMed: 10200226].

8. Martin R, Barr A, MacIntosh B, Smith R, Stevens T, Taves D, et al. Cerebral cortical processing of swallowing in older adults. Exp Brain Res. 2007;176(1):12-22. doi: 10.1007/s00221-006-0592-6. [PubMed: 16896984].

9. Shune SE, Moon JB, Goodman SS. The effects of age and preoral sensorimotor cues on anticipatory mouth movement during swallowing. J Speech Lang Hear Res. 2016;59(2):195-205. doi: 10.1044/2015_JSLHR-S15-0138. [PubMed: 26540553]. [PubMed Central: PMC4972007].

10. Braak H, Ghebremedhin E, Rub U, Bratzke H, Del Tredici K. Stages in the development of Parkinson's disease-related pathology. Cell Tissue Res. 2004;318(1):121-34. doi: 10.1007/s00441-004-0956-9. [PubMed: 15338272].

11. McHorney CA, Bricker DE, Kramer AE, Rosenbek JC, Robbins J, Chignell $\mathrm{KA}$, et al. The SWAL-QOL outcomes tool for oropharyngeal dysphagia in adults: I. Conceptual foundation and item development. Dysphagia. 2000;15(3):115-21. doi: 10.1007/s004550010012. [PubMed: 10839823].

12. Trail M. Neurorehabilitation in Parkinson's disease: an evidence-based treatment model. Slack Incorporated; 2008.

13. Baijens LW, Speyer R. Effects of therapy for dysphagia in Parkinson's disease: Systematic review. Dysphagia. 2009;24(1):91-102. doi: 10.1007/s00455-008-9180-1. [PubMed: 18931877].

14. van Hooren MR, Baijens LW, Voskuilen S, Oosterloo M, Kremer B. Treatment effects for dysphagia in Parkinson's disease: A systematic review. Parkinsonism Relat Disord. 2014;20(8):800-7. doi: 10.1016/j.parkreldis.2014.03.026. [PubMed: 24794097].

15. Regan J, Walshe M, Tobin WO. Immediate effects of thermal-tactile stimulation on timing of swallow in idiopathic Parkinson's disease. Dysphagia. 2010;25(3):207-15. doi: 10.1007/s00455-009-9244-x. [PubMed: 19707818].

16. Volonte MA, Porta M, Comi G. Clinical assessment of dysphagia in early phases of Parkinson's disease. Neurol Sci. 2002;23 Suppl 2:S1212. doi: 10.1007/s100720200099. [PubMed: 12548373].

17. Suntrup S, Teismann I, Bejer J, Suttrup I, Winkels M, Mehler D, et al. Evidence for adaptive cortical changes in swallowing in Parkinson's disease.Brain.2013;136(Pt3):726-38. doi:10.1093/brain/awt004. [PubMed: 23412935].

18. Chou KL, Evatt M, Hinson V, Kompoliti K. Sialorrhea in Parkinson's disease: A review. Mov Disord. 2007;22(16):2306-13. doi: 10.1002/mds.21646. [PubMed: 17659637].

19. Suttrup I, Warnecke T. Dysphagia in Parkinson's disease. Dysphagia. 2016;31(1):24-32. doi: 10.1007/s00455-015-9671-9. [PubMed: 26590572].

20. Gross RD, Atwood CW Jr, Ross SB, Eichhorn KA, Olszewski JW, Doyle PJ. The coordination of breathing and swallowing in Parkinson's disease. Dysphagia. 2008;23(2):136-45. doi: 10.1007/s00455-007-9113-4. [PubMed: 18027027].

21. Teismann IK, Steinstraeter O, Stoeckigt K, Suntrup S, Wollbrink A, Pantev $C$, et al. Functional oropharyngeal sensory disruption interferes with the cortical control of swallowing. BMC Neurosci.2007;8:62. doi: 10.1186/1471-2202-8-62. [PubMed: 17678546]. [PubMed Central: PMC1950520].

22. Monte FS, da Silva-Junior FP, Braga-Neto P, Nobre e Souza MA, de Bruin VM. Swallowing abnormalities and dyskinesia in Parkinson's disease. Mov Disord. 2005;20(4):457-62. doi: 10.1002/mds.20342. [PubMed: 15625689].

23. Grinberg LT, Rueb U, Alho AT, Heinsen H. Brainstem pathology and non-motor symptoms in PD. J Neurol Sci. 2010;289(1-2):81-8. doi: 10.1016/j.jns.2009.08.021. [PubMed: 19758601]. 
24. Groher ME, Crary MA. Dysphagia: Clinical management inadults and children. Elsevier Health Sciences; 2015.

25. Simons JA, Fietzek UM, Waldmann A, Warnecke T, Schuster T, Ceballos-Baumann AO. Development and validation of a new screening questionnaire for dysphagia in early stages of Parkinson's disease. Parkinsonism Relat Disord. 2014;20(9):992-8. doi: 10.1016/j.parkreldis.2014.06.008. [PubMed: 25012695].

26. Manor Y, Giladi N, Cohen A, Fliss DM, Cohen JT. Validation of a swallowing disturbance questionnaire for detecting dysphagia in patients with Parkinson's disease. Mov Disord. 2007;22(13):1917-21. doi: 10.1002/mds.21625. [PubMed: 17588237].

27. Ciucci MR, Grant LM, Rajamanickam ESP, Hilby BL, Blue KV, Jones CA, et al. Early identification and treatment of communication and swallowing deficits in Parkinson disease. Seminars in speech and language. Thieme Medical Publishers; 2013.

28. Felix VN, Correa SM, Soares RJ. A therapeutic maneuver for oropharyngeal dysphagia in patients with Parkinson's disease. Clinics (Sao Paulo). 2008;63(5):661-6. doi: 10.1590/s1807-59322008000500015. [PubMed: 18925327]. [PubMed Central: PMC2664725].

29. Nagaya M, Kachi T, Yamada T. Effect of swallowing training on swallowing disorders in Parkinson's disease. Scand $J$ Rehabil Med. 2000;32(1):11-5. doi: 10.1080/003655000750045677. [PubMed: 10782935].

30. Van Lieshout PH, Steele CM, Lang AE. Tongue control for swallowing in Parkinson's disease: Effects of age, rate, and stimulus consistency. Mov Disord. 2011;26(9):1725-9. doi: 10.1002/mds.23690. [PubMed: 21542018].

31. Logemann JA, Gensler G, Robbins J, Lindblad AS, Brandt D, Hind JA, et al. A randomized study of three interventions for aspiration of thin liquids in patients with dementia or Parkinson's disease.J Speech Lang Hear Res. 2008;51(1):173-83. doi: 10.1044/1092-4388(2008/013). [PubMed: 18230864]. [PubMed Central: PMC2894528].

32. Pisegna JM, Kaneoka A, Pearson WG Jr, Kumar S, Langmore SE. Effects of non-invasive brain stimulation on post-stroke dysphagia: A systematic review and meta-analysis of randomized controlled trials. Clin Neurophysiol. 2016;127(1):956-68. doi: 10.1016/j.clinph.2015.04.069. [PubMed: 26070517]. [PubMed Central: PMC5326549].

33. Troche MS, Okun MS, Rosenbek JC, Musson N, Fernandez HH, Rodriguez R, et al. Aspiration and swallowing in Parkinson disease and rehabilitation with EMST: A randomized trial. Neurology. 2010;75(21):1912-9. doi: 10.1212/WNL.ob013e3181fefi15. [PubMed: 21098406]. [PubMed Central: PMC2995389].

34. Manor Y, Mootanah R, Freud D, Giladi N, Cohen JT. Video-assisted swallowing therapy for patients with Parkinson's disease. Parkinsonism Relat Disord. 2013;19(2):207-11. doi: 10.1016/j.parkreldis.2012.10.004. [PubMed: 23131836]

35. Willig TN, Paulus J, Lacau Saint Guily J, Beon C, Navarro J. Swallowing problems in neuromuscular disorders. Arch Phys Med Rehabil. 1994;75(11):1175-81. doi: 10.1016/0003-9993(94)90001-9. [PubMed: 7979925].

36. Baijens LW, Speyer R, Passos VL, Pilz W, van der Kruis J, Haarmans $S$, et al. Surface electrical stimulation in dysphagic Parkinson patients: A randomized clinical trial. Laryngoscope. 2013;123(11):E38-44. doi: 10.1002/lary.24119. [PubMed: 23595549].

37. Heijnen BJ, Speyer R, Baijens LW, Bogaardt HC. Neuromuscular electrical stimulation versus traditional therapy in patients with Parkinson's disease and oropharyngeal dysphagia: Effects on quality of life. Dysphagia. 2012;27(3):336-45. doi:10.1007/s00455-011-9371-z. [PubMed: 22081122]. [PubMed Central: PMC3417093].

38. Yang EJ, Baek SR, Shin J, Lim JY, Jang HJ, Kim YK, et al. Effects of transcranial direct current stimulation (tDCS) on post-stroke dysphagia. Restor Neurol Neurosci. 2012;30(4):303-11. doi: 10.3233/RNN-2012-110213. [PubMed: 22572022].

39. Kumar S, Wagner CW, Frayne C, Zhu L, Selim M, Feng W, et al. Noninvasive brain stimulation may improve stroke-related dysphagia: A pilot study. Stroke. 2011;42(4):1035-40. doi: 10.1161/STROKEAHA.110.602128. [PubMed: 21441148]. [PubMed Central: PMC3116809].

40. Shigematsu T, Fujishima I, Ohno K. Transcranial direct current stimulation improves swallowing function in stroke patients. Neurorehabil Neural Repair. 2013;27(4):363-9. doi: 10.1177/1545968312474116. [PubMed: 23392916]. 